\title{
Kinderfeste hinter Stacheldraht: Die Entwicklung der NPD in Mecklenburg-Vorpommern nach der Landtagswahl 2011
}

\author{
Marc Brandstetter*
}

\section{Neuordnung der rechtsextremistischen Parteienlandschaft}

In den letzten Jahren durchlief die parteipolitisch organisierte extremistische Rechte einen tiefgreifenden Umstrukturierungsprozess. Die DVU, einst von dem mittlerweile verstorbenen Gerhard Frey, einem vermögenden Rechtsanwalt und Verleger aus München gegründet, erklärte im Frühjahr 2012 ihre Auflösung. Zur Jahreswende 2010 auf 2011 hatten die damaligen Parteivorsitzenden von NPD und DVU, Udo Voigt und Matthias Faust, einen so genannten Verschmelzungsvertrag unterschrieben: Eine vereinigte Rechte sollte die Bundesrepublik das Fürchten lehren. Die wenigen Landesverbände, die sich zuvor dieser Vereinigung widersetzt hatten, waren mit ihrer Klage vor dem Landgericht München gescheitert und hatten daraufhin resigniert aufgegeben. ${ }^{1}$

Ihre erfolgreichsten Tage hatte die DVU bereits lange hinter sich. 1998 fuhr sie mit einer großangelegten Materialschlacht bei den Landtagswahlen in Sachsen-Anhalt 12,9 Prozent ein, bis heute das höchste Ergebnis einer rechtsgerichteten Partei seit 1949. ${ }^{2}$ Danach zeigte der Trend - mit kleineren Unterbrechungen wie die Einzüge in den Brandenburgischen Landtag 1999 und $2004^{3}$ - nach unten. Außerdem hatte die Organisation mit sinkenden Mitgliederzahlen zu kämpfen. ${ }^{4}$ Anstatt Aufbruchstimmung zu verbreiten, manövrierte Faust, der den Parteivorsitz von Frey Anfang 2009 übernommen hatte, die DVU weiter in die Krise. ${ }^{5}$ Die Spitzenfunktionäre hofften, der Zusammenschluss von DVU und NPD würde beide Parteien aus ihrer Lethargie herausreißen. Doch die Fusion war nicht der anvisierte große Wurf. Die NPD bediente sich aus der Konkursmasse der DVU, die nüchtern betrach-

* Marc Brandstetter ist Redakteur der Internet- und Beteiligungskampagne „Endstation Rechts“.

1 Vgl. Homepage der DVU-Berlin, Das Ende der DVU. Der letzte Eintrag, 26. Mai 2012, http:// www.dvu-berlin.info/aktuelles.html (Abruf am 1. März 2013).

2 Vgl. Stefan Schieren, Die Landtagswahl in Sachsen-Anhalt vom 26. April 1998: „Magdeburger Modell“ mit einigen Mängeln, in: ZParl, 30. Jg. (1999), H. 1, S. 56 - 78, S. 62 ff.; siehe zur DVU auch Everhard Holtmann, Die angepassten Provokateure. Aufstieg und Niedergang der rechtsextremen DVU als Protestpartei im polarisierten Parteiensystem Sachsen-Anhalts, Opladen 2001.

3 Vgl. Karl Schmitt, Die Landtagswahlen in Brandenburg und Thüringen vom 5. und 12. September 1999: Landespolitische Entscheidungen im Schlagschatten der Bundespolitik, in: ZParl, 31. Jg. (2000), H. 1, S. 43 - 68, S. 55 f.; Oskar Niedermayer, Die brandenburgische Landtagswahl vom 19. September 2004: Reaktionen der Wähler auf Hartz IV, in: ZParl, 36. Jg. (2005), H. 1, S. 64 -80 , S. $71 \mathrm{ff}$.

4 Vgl. zum Beispiel Bundesministerium des Innern, Rechtsradikale Parteien in Deutschland: Entwicklung der Mitgliederzahlen von NPD, DVU und Republikanern von 2005 bis 2011, Veröffentlichung im Juli 2012, http://de.statista.com/statistik/daten/studie/4760/umfrage/entwicklungder-mitgliederzahlen-von-rechtsradikalen-parteien/ (Abruf am 5. März 2013).

5 Vgl. Marc Brandstetter, Mit dem Rücken zur Wand. Die Krise der rechtsextremistischen Parteien, in: Deutschland Archiv, 43. Jg. (2010), H. 4, S. 595 - 598, S. 596. 
tet ohnehin gering war. Eine Vereinigung auf Augenhöhe - wie beide Partner stets versicherten - gab es nicht. ${ }^{6}$

Mit der Partei „Die Rechte“ (DR) hat dafür kürzlich eine neue Konkurrenz die politische Bühne betreten. Am Pfingstsonntag 2012 von dem bekannten Neonazi Christian Worch in „kleinem geschlossenen Rahmen“ gegründet, sei sie auf den „Trümmern der DVU“ aufgebaut, wie es in einer Stellungnahme auf der DR-Internetseite vom 8. Juni hieß. In ihrem Parteiprogramm bekennt sich die Splitterorganisation, die nach eigenen Angaben knapp 300 Anhänger umfasst, „vollinhaltlich und ohne jeden Vorbehalt zur freiheitlich-demokratischen Grundordnung, wie sie im Grundgesetz für die Bundesrepublik Deutschland verfasst ist "7. Tatsächlich steht bereits die personelle Zusammensetzung der „Rechten“ im Gegensatz zu ihrer angeblichen ideologischen Mäßigung, denn die neue Partei fungiert als Sammelbecken für verbotene Neonazi-Kameradschaften. ${ }^{8}$

Auch das einstige Flaggschiff der rechtsextremen Parteien, die NPD, hat derzeit Schlagseite. Bei den zurückliegenden Landtagswahlen im Saarland, in Schleswig-Holstein, Nordrhein-Westfalen und Niedersachsen büßte sie jeweils rund ein Drittel ihrer Anhänger ein. ${ }^{9}$ Nur im Saarland übersprang die Partei die wichtige Marke von einem Prozent, um in den Genuss staatlicher Gelder aus der Parteienteilfinanzierung zu kommen. ${ }^{10}$ Da sie zusätzlich mit ihrer Klage vor dem Bundesverwaltungsgericht teilweise scheiterte, muss sie rund 1,3 Millionen Euro Strafgelder, die aus der Abgabe falscher Rechenschaftsberichte resultieren, an die Bundestagsverwaltung zahlen ${ }^{11}$ - ein schwerer Schlag für die ohnehin ständig in Geldnot befindliche Partei, der ihre politische Handlungsfähigkeit weiter beschneidet. Die „gemäßigtere" Ausrichtung des neuen Bundesvorsitzenden Holger Apfel verprellt darüber hinaus „radikalere“ Parteianhänger: Zwei Flügel stehen sich nahezu unversöhnlich gegenüber, die NPD ist tief gespalten. Die Mitglieder laufen ihr davon - zuletzt waren es noch 5.900, von denen aber nur 3.000 politisch aktiv seien, wie Apfel im Spiegel eingestehen musste. ${ }^{12}$ Der Beschluss der Bundesländer, vor dem Bundesverfassungsgericht einen Antrag

6 Vgl. Lars Normann, Vorstandswechsel, Fusion und Ende der „Phantompartei“ DVU, in: Uwe Backes / Alexander Gallus / Eckhard Jesse (Hrsg.), Jahrbuch Extremismus \& Demokratie, Bd. 23, Baden-Baden 2011, S. 165 - 184, S. 180 ff.

7 Zitiert nach Die Rechte, Programm der Partei Die Rechte, S. 1, http://worch.info/die-rechte/pdf/ Parteiprogramm.pdf (Abruf am 5. März 2013).

8 Vgl. Marc Brandstetter, „Die Rechte“ in Bewegung, in: Blätter für deutsche und internationale Politik 2013, H. 2, S. 13 - 16.

9 Vgl. zu den Landtagswahlen: Jürgen R. Winkler, Die saarländische Landtagswahl vom 25. März 2012: Von Jamaika zur Großen Koalition, in: ZParl, 43. Jg. (2012), H. 3, S. 507 - 524, S. 513 ff.; Patrick Horst, Die schleswig-holsteinische Landtagswahl vom 6. Mai 2012: SPD, Grüne und SSW bilden erste Dänen-Ampel, in: ebenda, S. 524 - 543, S. 533; Stefan Bajohr, Die nordrheinwestfälische Landtagswahl vom 13. Mai 2012: Von der Minderheit zur Mehrheit, in: ebenda, S. 543 - 563, S. 555 f.; Niedersächsische Landeswahlleiterin, Amtliches Endergebnis der Wahl des Niedersächsischen Landtages der 17. Wahlperiode am 20. Januar 2013, S. 93, http://www.landeswahlleiter.niedersachsen.de/portal/live.php?navigation_id=6743\&article_id=100052\&_psmand=21 (Abruf am 5. März 2013).

10 Vgl. Jürgen R. Winkler, a.a.O. (Fn. 9), S. 516.

11 Vgl. BVerwG 6 C 32.11 - Urteil vom 12. Dezember 2012.

12 Vgl. Jürgen Dahlkamp / Gunther Latsch / Maximilian Popp / Sven Röbel / Holger Stark / Andreas Wassermann / Steffen Winter, Eine unerträgliche Partei, in: Der Spiegel vom 13. Februar 2012, S. $32-41$. 
auf ein Verbot der rechtsextremistischen Partei zu stellen ${ }^{13}$, bedeutet eine ernsthafte Gefahr für die Existenz der NPD. Ihr 50-jähriges Bestehen 2014 wird sie möglicherweise nicht mehr erleben. In Mecklenburg-Vorpommern hingegen ist die Situation eine andere: Eine Spaltung wie auf Bundesebene ist nicht zu beobachten, die Partei steht „voll im Saft“ und treibt ihre „nationale Graswurzelrevolution“ mit Nachdruck voran.

\section{Landtagswahl 2011}

Frühzeitig hatte die NPD die Weichen für den Wiedereinzug in den Landtag von Mecklenburg-Vorpommern gestellt. Im Gegensatz zu den anderen Parteien fuhr sie ihre Aktivitäten kaum zurück und befand sich fast fünf Jahre lang in einer Art „Dauerwahlkampf“, dessen Ziel die weitere strukturelle und dauerhafte Verwurzelung in der Gesellschaft des Ostseebundeslandes war und ist. ${ }^{14}$ Die Voraussetzungen waren günstig: Die zurückliegenden Wahlergebnisse - Kommunalwahl 2009: 3,2 Prozent, Bundestagswahl 2009: 3,3 Prozent - deuteten ihr Stammwählerpotential an.

Die von ihrem Fraktionsvorsitzenden Udo Pastörs in den Wahlkampf geführte Partei setzte inhaltlich auf eine Kombination aus Fremdenfeindlichkeit und sozialpolitischen Themen. In ihr Visier gerieten insbesondere die polnischen Nachbarn. Entsprechende Plakate mit provokanten Aussagen wie „Polen offen. Arbeit futsch. Auto weg! - Arbeitsplätze sichern - Grenzen dicht!“ oder „Atomtod aus Polen stoppen“ wurden vor allem in den Grenzregionen geklebt. Diese Landstriche in Vorpommern gehören traditionell zu den Hochburgen der rechtsextremistischen Partei. ${ }^{15}$ Die Strategen wussten um ihre Chancen ebenso wie um die Parolen, die dort greifen würden. Kein Wunder, dass das aus anderen Wahlkämpfen bekannte Motiv „Kriminelle Ausländer raus“ zum Einsatz kam. Außerdem bewiesen die NPD-Wahlkämpfer Gespür für populistisch ausschlachtbare Themen. Wo auch immer das örtliche Klima, beispielsweise durch die drohende Schließung einer größeren Fabrik, aufgeheizt war - die NPD war vor Ort. Das Auftreten der Führungsriege strotzte vor Selbstbewusstsein. In aggressiver Manier tauchte sie bei Veranstaltungen des politischen Gegners auf, um diesen verbal zu attackieren. Im Gepäck hatten die Aktivisten stets eine Videokamera, um ihre Auftritte auf den bekannten der Partei nahestehenden Internetportalen, zu verbreiten. ${ }^{16}$

Die Mühe lohnte. Am Wahltag entschieden sich 6,0 Prozent der Wahlberechtigten für die Rechtsextremisten. Dies bedeutete zwar den Verlust von 1,3 Prozentpunkten, sicherte der Nordost-NPD aber den bundesweiten Spitzenplatz. In Sachsen hatte der dortige (,gemäßigtere“) Landesverband zwei Jahre zuvor „nur“ 5,6 Prozent gewonnen. Vor allem „Protestwähler" gingen von der Fahne, 18.000 Menschen, die 2006 noch für die NPD gestimmt

13 Vgl. auch den Beitrag von Philipp Erbentraut in diesem Heft der ZParl.

14 Vgl. Steffen Schoon, Gefestigt und begrenzt - die NPD in Mecklenburg-Vorpommern. Eine Betrachtung anhand der Landtagswahl 2011, 6. Januar 2012, http://www.bpb.de/ themen/7KZRPJ,0,Gefestigt_und_begrenzt\%96_die_NPD_in_MecklenburgVorpommern.html (Abruf am 3. Januar 2012).

15 Vgl. ebenda.

16 Vgl. Martin Koschkar / Steffen Schoon, Die mecklenburg-vorpommersche Landtagswahl vom 4. September 2011: Bestätigung der Großen Koalition mit sozialdemokratischem Zugewinn, in: ZParl, 43. Jg. (2012), H. 1, S. 3 - 18, S. 7. 
hatten, enthielten sich der Stimme. Ein Wähleraustausch mit den demokratischen Parteien fand indes nicht statt. ${ }^{17}$

Wenngleich der Zuspruch für die NPD in allen Landesteilen - außer dem Wahlkreis Uecker-Randow (plus 0,4 Prozentpunkte) - rückläufig war, veränderten sich ihre Hochburgen im Vergleich zu 2006 kaum. Sie lagen und liegen in den dünn besiedelten Gebieten im Osten des Bundeslandes, in Vorpommern. Während sie in Mecklenburg durchschnittlich 5,3 Prozent gewann, waren es hier 8,4 Prozent. ${ }^{18}$ Auch im westlichen Teil des Landes hat die NPD ihre Schwerpunktregionen. Dies gilt im Besonderen für den Wahlkreis Ludwigslust I, wo sich 8,3 Prozent der Wähler für sie entschieden. ${ }^{19}$ Dort, wo die NPD traditionell stark ist, bleibt sie es; neue Hochburgen gewinnt sie aber nicht hinzu.

Grund, in übermäßigen Jubel auszubrechen, bot dieses Ergebnis der Partei also nicht. In einer Stellungnahme griff die NPD-Führung auf ihre typische kämpferische Rhetorik zurück und strich gleichzeitig die Anstrengungen ihrer Anhänger heraus. Der erneute Einzug ins Schweriner Schloss sei ein „Kraftakt“ gewesen. Gleichzeitig geißelte sie die Kampagne der demokratischen Kräfte des Bundeslandes, die bis zuletzt versucht hatten, die NPD unter die Fünfprozenthürde zu drücken: „Diese feinen Damen und Herren haben mit der Demokratie (= Volksherrschaft!) genauso viel am Hut wie skrupellose Spekulanten mit sozialer Verantwortung." 20

\section{Nach der Aufdeckung des "Nationalsozialistischen Untergrunds"}

Wie die Bundespartei wurde der Landesverband Mecklenburg-Vorpommern von der Aufdeckung der rassistisch motivierten Mordserie der rechtsterroristischen Gruppierung „Nationalsozialistischer Untergrund“ (NSU) im November 2011 unvorbereitet getroffen. Bald diskutierte die Öffentlichkeit wieder über ein Verbot der NPD. In Umfragen sprach sich eine breite Mehrheit der Befragten für ein rigoroses Vorgehen des Staates aus. ${ }^{21}$ Ein erstes Verbotsverfahren war 2003 gescheitert. ${ }^{22}$ Damals sahen drei der sieben Richter des 2. Senats des Bundesverfassungsgerichtes in der Einschleusung von Informanten des Verfassungsschutzes in die NPD-Führungsgremien ein „nicht behebbares Verfahrenshindernis“. Die Kammer fällte eine Prozess- und keine Sachentscheidung, der NPD wurde kein „Persilschein“ ausgestell $^{23}$, obgleich sie seither stetig versucht, diesen Eindruck zu erwecken. Dennoch ist nicht von der Hand zu weisen, dass die „Niederlage“ der Bundesrepublik der NPD einigen

17 Vgl. Infratest dimap (Hrsg.), Mecklenburg-Vorpommern 2011. Eine Analyse der Wahl vom 4. September 2011, Berlin 2011, S. 10; Martin Koschkar / Steffen Schoon, a.a.O. (Fn. 16), S. 8 f.

18 Vgl. Martin Koschkar / Steffen Schoon, a.a.O. (Fn. 16), S. 9.

19 Vgl. Steffen Schoon, a.a.O. (Fn. 14).

20 Vgl. Pressemitteilung des NPD-Landesverbandes Mecklenburg-Vorpommern, NPD erkämpft den Wiedereinzug in den Landtag, 5. September 2011, http://www.npd-mv.de/index.php?com=news \&view=article\&id=2505\&mid=7 (Abruf am 5. März 2013).

21 Vgl. ZDF-Politikbarometer, Piraten deutlich im Aufwind - FDP bleibt im Keller, 30. März 2012, http://www.zdf.de/ZDF/zdfportal/web/ZDF.de/Politbarometer/2942200/6628476/b4edb2/Piraten-deutlich-im-Aufwind---FDP-bleibt-im-Keller.html (Abruf am 15. Juli 2012).

22 Vgl. Lars Flemming, Das NPD-Verbotsverfahren. Vom „Aufstand der Anständigen“ zum „Aufstand der Unfähigen“, Baden-Baden 2004.

23 Vgl. Eckhard Jesse, Der gescheiterte Verbotsantrag gegen die NPD. Die streitbare Demokratie ist beschädigt worden, in: PVS, 44. Jg. (2003), H. 3, S. 292 - 301, S. 294. 
Auftrieb bescherte, da die randständige Partei zurück in die öffentliche Aufmerksamkeit katapultiert wurde. Selbst wenn für die Rechtsextremisten die Verfahrenseinstellung problematisch war - sie war nicht den „Märtyrertod“ gestorben, zudem vergiftete die Ungewissheit der Mitglieder, wer was über wen wusste, das innerparteiliche Klima -, schlug sie politisches Kapital aus dem Versagen der politischen Klasse. Sie fühlte sich „unverbietbar“; aus der systematischen Integration von Neonazis, die die NPD-Strategen seit Beginn der neunziger Jahre weitgehend geräuschlos betrieben, wurde ein offenes Werben: Die „Volksfront von Rechts“ war geboren. Die frischen Kräfte sorgten für einen weiteren Radikalisierungsschub.

\section{Die Hardliner unter den Radikalen}

Weitreichende Folgen hatte diese Vorgehensweise in Mecklenburg-Vorpommern, wo die NPD durch eine Eintrittswelle ehemals „freier“ Kameradschaftsaktivisten in den Monaten vor der Landtagswahl 2006 erst zu einer ernst zu nehmenden politischen Kraft geworden war. Vorher spielte sie an der Ostsee keine Rolle, sogar eine Spaltung des Landesverbandes, die sie einige ihrer wenigen Führungskader kostete, musste verkraftet werden. Bis 2005 dominierten die Neonazi-Kameradschaften die extremistische Rechte in diesem Bundesland. ${ }^{24}$ Der dann erfolgte Zusammenschluss der beiden Flügel der „Bewegung“ sorgte für eine Sonderstellung dieser Gliederung: Nur in wenigen NPD-Landesverbänden ist die Verschmelzung zwischen klassischem Partei- und radikalem Neonazimilieu soweit fortgeschritten wie in Mecklenburg-Vorpommern. So überrascht es kaum, dass die Mannschaft um den Landesvorsitzenden Stefan Köster zu den radikalsten NPD-Verbänden gehört.

Eine Blamage vor dem Bundesverfassungsgericht wie beim ersten Verbotsverfahren wollte die Politik von vornherein ausschließen und machte Nägel mit Köpfen. Die Innenminister von Bund und Ländern beschlossen, ihre „V-Leute“ in den NPD-Führungsetagen „abzuschalten“. Außerdem sollten die zuständigen Stellen mit der Beweissammlung beginnen. Wenngleich immer wieder skeptische Stimmen - besonders aus den Reihen der Union und der FDP - laut wurden, trugen die Verfassungsschutzämter des Bundes und der Länder doch etliches Beweismaterial gegen die NPD zusammen, das mehrheitlich aus öffentlichen Quellen stammt und nicht mit nachrichtendienstlichen Mitteln gewonnen wurde. ${ }^{25}$ Im Dezember des vergangenen Jahres sprachen sich die Innenminister der Länder auf ihrer Konferenz in Rostock-Warnemünde einstimmig für ein neues Verbotsverfahren aus. Die Ministerpräsidentenkonferenz folgte dem Votum ebenfalls einstimmig. Daraufhin beschloss der Bundesrat am 14. Dezember 2012 mit großer Mehrheit - nur Hessen enthielt sich der Stimme -, erneut ein Verbot der NPD beim Bundesverfassungsgericht zu beantragen. ${ }^{26}$

Die NPD reagiert mit einer zweigleisigen Strategie auf die Bedrohungslage: Einerseits versuchten die führenden Kader mit wortgewaltigen Erklärungen, einen möglichst breiten

24 Vgl. Mathias Brodkorb / Volker Schlotmann (Hrsg.), Provokation als Prinzip. Die NPD im Landtag von Mecklenburg-Vorpommern, Schwerin 2008.

25 Vgl. Frank Jansen, Über 3000 Beweise für verfassungsfeindliche Haltung der NPD, in: Der Tagesspiegel vom 24. September 2012, http://www.tagesspiegel.de/politik/rechtsextremismus/moegliches-verbotsverfahren-ueber-3000-belege-fuer-verfassungsfeindliche-haltung-der-npd/7173414. html (Abruf am 1. Oktober 2012).

26 Vgl. „Zweiter Anlauf für NPD-Verbot“, in: SZ vom 15. Dezember 2012, S. 6. 
Trennstrich zum gewaltbereiten rechtsextremistischen Milieu zu ziehen, andererseits mäßigten sie ihre öffentlichen Auftritte. Führende Neonazis wie Martin Wiese oder Karl-Heinz Hoffmann verloren ihr Rederecht auf Parteidemonstrationen. Die beiden zählen zu den militantesten Figuren der Szene, der eine - Wiese -, weil er für einen geplanten Sprengstoffanschlag auf das jüdische Zentrum in München zu sieben Jahren Haft verurteilt worden war, der andere - Hoffmann -, weil er in den siebziger Jahren die später verbotene paramilitärische „Wehrsportgruppe Hoffmann“ angeführt hatte, aus deren Umfeld zahlreiche Gewalttaten erfolgten.

Von der neuen Ausrichtung waren die (parlamentarischen) Initiativen der Schweriner Fraktion betroffen. In den Monaten nach dem Bekanntwerden der Existenz der „Zwickauer Zelle“ agierte die NPD im Plenum verhältnismäßig zahm. Zwar waren Ordnungsrufe gegen ihre Abgeordneten weiterhin an der Tagesordnung, doch die Anträge und Reden der fünf NPD-Abgeordneten beinhalteten weniger nationalistische, rassistische, fremdenfeindliche oder chauvinistische Aussagen als vormals. Unsicherheit kennzeichnete das Verhalten der rechtsextremistischen Fraktion. Aus der unglaublichen Serie von Fehlern, Pannen, Versäumnissen und teilweise gezielten Vertuschungsversuchen der Behörden konstruierte sie - ganz ihrer typischen Denkweise verhaftet - abstruse Verschwörungstheorien, in denen der Verfassungsschutz die Hauptrolle übernahm. Nach ihrer Lesart hatte dieser den NSU gesteuert, um den „nationalen Widerstand“ zu diskreditieren. Wieder einmal sang die NPD das Lied des Opfers staatlicher Willkür, das sie so vortrefflich beherrscht. Abwiegeln, ablenken und andere verdächtigen, lautete die Devise. ${ }^{27}$

Von dieser taktischen Zurückhaltung war in ihrer Fraktions-Postille, dem „Ordnungsruf“, nicht viel zu spüren. Dort nahmen die Autoren kein Blatt vor den Mund und hetzten mit den bekannten derben Worten gegen „Ausländer“ und das (politische) Establishment. ${ }^{28}$ Ohnehin wich die Mäßigung der Parteikader bald ihrer gewohnten Vorgehensweise. Ihre Initiativen im Landtag artikulierte die Fraktion in schärferem Ton, auf die Tagesordnung schafften es vermehrt Anträge, die in einem ideologischen Licht schienen. Immer wieder war das Landtagspräsidium gezwungen, die NPD-Abgeordneten mit Sanktionen zu maßregeln. Besonders unter den beiden Fraktionsspitzen Udo Pastörs und Stefan Köster scheint es eine Art Wettlauf zu geben, wer innerhalb kürzester Zeit die meisten Tadel sammeln könne. Gleich mehrere Male erfolgte der Wortentzug oder sogar der Ausschluss aus der laufenden Sitzung beziehungsweise für kommende Beratungen.

Unter den fünf NPD-Abgeordneten im neuen Landtag fand sich mit David Petereit (Jahrgang 1981), dem ehemaligen Wahlkreismitarbeiter des nicht mehr angetretenen Birger Lüssow, ein neues Gesicht. Ansonsten veränderte sich die Fraktion nicht. Mit ihrem Chef Udo Pastörs, dem Landesvorsitzenden Stefan Köster, dem aus Vorpommern stammenden Kameradschaftsaktivisten Tino Müller und dem umtriebigen Multifunktionär Michael Andrejewski blieb alles beim Alten.

Mit nun fünf Abgeordneten fallen die finanziellen Zuwendungen des Landes Mecklenburg-Vorpommern geringer aus als in der zurückliegenden Wahlperiode. Während die NPD-

27 Vgl. Marc Brandstetter, 6. Landtagssitzung: Von Wattestäbchen und Legionen von V-Leuten, 15. Dezember 2011, http://www.endstation-rechts.de/index.php?option=com_k2\&view=item \&id=6707:6-landtagssitzung\&Itemid=358 (Abruf am 1. Oktober 2012).

28 Vgl. Ordnungsruf, in: Mitteilungsblatt der NPD-Fraktion in Mecklenburg-Vorpommern, Ausgabe 12/2012, Schwerin 2012. 
Abgeordneten zwischen 2006 und 2011 Entschädigungen, steuerfreie Kostenpauschalen, Aufwendungen für die Wahlkreismitarbeiter, Fraktionszuschüsse und den Dienstwagen des Vorsitzenden in Höhe von rund 1,4 Millionen Euro pro Jahr kassierten, fließen in den nächsten fünf Jahren circa eine Million Euro auf die Konten der Rechtsextremisten. ${ }^{29}$

Nicht mehr in die Volksvertretung schafften es Lüssow und Raimund Borrmann, der in der 5. Wahlperiode vor allem mit merkwürdigem Verhalten wie der Weigerung, die gebräuchliche Parlamentsanrede „Frau Präsidentin, meine Damen und Herren“ zu verwenden, von sich reden machte. Stattdessen benutzte er die Formel „Voice of the blood. Stimme des Blutes", was ihm zahlreiche Ordnungsrufe einbrachte. Bei NPD-Kinderfesten trat Borrmann als Clown auf - diese Rolle schien er auch im Plenum übernommen zu haben.

Petereit hingegen ist aus anderem Holz geschnitzt. Mit ihm als neuen Abgeordneten tritt die Fraktion homogener auf als im letzten Landtag. "Totalausfälle“ wie die Hinterbänkler Lüssow und Borrmann hat sie keine mehr in ihren Reihen. Petereit, langjähriger Führungskader der mittlerweile verbotenen „Mecklenburgischen Aktionsfront“ (MAF), der verbotenen „Heimattreuen Deutschen Jugend“ (HDJ) sowie der ebenfalls verbotenen „Hilfsorganisation für nationale politische Gefangene und deren Angehörige“ (HNG), ist gelernter Rechtsanwaltsfachangestellter und studierte an der Rostocker Universität Rechtswissenschaften. ${ }^{30}$ Er gilt als beinharter Überzeugungstäter. Im Parlament redet er zu Themen wie „Bildung“, „Verfassungsschutz“ oder „linksextremer“ Gewalt. Meist liest er seine ausformulierten Reden vom Blatt ab. Dabei ist ein gewisser süffisanter Unterton, der die Geringschätzung des Parlaments ausdrückt, sein stetiger Begleiter. Richtig in Fahrt kommt Petereit hingegen, wenn er seine vorgezeichnete Linie verlässt und weitgehend frei spricht. Dann zeigt sich seine Erfahrung aus den Reden vor Gesinnungsgenossen. Unverblümt drückt er seine Ablehnung des demokratischen Systems, dessen Institutionen und Parteien aus. Mit ihm ist der NPD auch der Brückenschlag zwischen Mecklenburg und Vorpommern gelungen. Lag in der vorangegangenen Wahlperiode die Mitte des Bundeslandes parlamentarisch brach, beackert der 31-Jährige nun diese Gebiete.

Die auf Petereit registrierte Internetseite „MUPinfo“ gehört zu den wichtigsten Kommunikations- und Informationsinstrumenten der extremistischen Rechten im Nordosten. Bereits im Wahlkampf griff die NPD-Führung gern und häufig auf das Portal zurück, um ihre Propaganda zu verbreiten. Durch die zeitweilige Abschaltung (ehemals) einflussreicher Szenemedien wie „Altermedia“, „Thiazi-Forum“ oder „DeutschlandEcho“ ist seine Bedeutung nochmals gestiegen. Zugleich ist durch den Einzug Petereits ins Landesparlament die - ohnehin enge - Verflechtung mit der freien Kameradschaftsszene noch dichter geworden.

Petereit steht für einen Generationswechsel, den die NPD seit der Landtagswahl schrittweise vorantreibt. In seinem Schatten drängen einige Jungfunktionäre wie Tino Streif, Frank Klawitter und Hannes Welchar ins Rampenlicht. Streif verantwortet nicht nur die Webseite des „Thinghauses“, sondern scheint nach der Inhaftierung Sven Krügers (siehe unten) die Fäden in Jamel und Umgebung in die Hand genommen zu haben. Nicht zufällig unternimmt mittlerweile die bis dato unbedeutende NPD-Jugendorganisation Junge Nationaldemokraten (JN) erste zaghafte Gehversuche. Über die internen Veranstaltungen und Schulungen hinaus, die oft im "Thinghaus“ stattfinden, marschierte die JN erstmals zu einer

29 Vgl. Parlamentsdatenbank des Landes Mecklenburg-Vorpommern; eigene Berechnung.

30 Vgl. Landtag Mecklenburg-Vorpommern (Hrsg.), Handbuch. Der Landtag Mecklenburg-Vorpommern. 6. Wahlperiode 2011 - 2016, Schwerin 2012, S. 53. 
Demonstration am 20. Oktober unter dem Motto „Wir wollen leben“ als Teil der auch in Mecklenburg-Vorpommern von der extremen Rechten rezipierten „Volkstod-Kampagne“ auf. Dass der NPD-Nachwuchs genau dieses Thema ausgewählt hatte, ist beabsichtigt, denn mit dem Bundesvorstandsmitglied Sebastian Richter hat kürzlich ein JN-Führungskader seinen Wohnsitz nach Mecklenburg-Vorpommern verlegt, der aus dem brandenburgischen „Spreelichter"-Milieu stammt. ${ }^{31}$ Geführt wird der Parteinachwuchs seit 2011 von Alf Börm, dem Sohn des vorbestraften Rechtsextremisten Manfred Börm, der lange dem NPD-Ordnerdienst vorstand. Wie Streif studiert(e) Börm Maschinenbau.

Auch auf der Straße zeigte die Ostsee-NPD Präsenz. Am 1. Mai 2012 meldete sie eine Demonstration in Neubrandenburg an, zu der sie rund 300 Aktivisten - vor allem aus der Freien Kameradschaftsszene - mobilisieren konnte. Ihr Fraktionschef Pastörs sollte am selben Tag den Part des Hauptredners bei einer Kundgebung in Neumünster übernehmen, die als Wahlkampfhöhepunkt und -abschluss zur Landtagswahl in Schleswig-Holstein konzipiert war. Doch dazu kam es erst gar nicht: Da Pastörs mit seinen 120 Anhängern eine Bahnstation vor dem eigentlichen Sammelpunkt ausgestiegen war und der Demonstrationszug somit gegen behördliche Auflagen verstoßen hatte, wurde der Aufmarsch von einem Polizeiaufgebot festgesetzt. Die Rechtsextremisten weigerten sich, auf die genehmigte Route zurückzukehren, Pastörs lieferte sich ein heftiges Wortgefecht mit dem Einsatzleiter der Polizei, in dem er drohte, „man könne die Situation“ eskalieren lassen. Die Polizeiführung ließ sich nicht einschüchtern und griff mit harter Hand durch. Pastörs wurde gemeinsam mit den anderen Demonstrationsteilnehmern, zu denen die beiden schleswig-holsteinischen Spitzenkader Jens Lütke und Ingo Stawitz gehörten, unter dem Jubel der Gegendemonstranten abgeführt und zur Feststellung der Identität auf die Wache gebracht. ${ }^{32}$

\section{Die Verwurzelung in den lokalen Gesellschaften als Ziel - die NPD im "Dauerwahlkampf"}

Auch im ersten Jahr nach der Landtagswahl blieb die NPD ihrer gewohnten Strategie treu und versuchte, mit einer Art „Dauerwahlkampf“ im öffentlichen Leben präsent zu sein. Indem sie suggeriert, sie gehöre zur Gesellschaft, sei eine „normale“ Partei wie die anderen, will sie die gesellschaftliche Ausgrenzung unterlaufen. Zu diesem Zweck meldete die Fraktion eine Reihe von Infoständen an, deren Schwerpunkt in der Hauptstadt Schwerin lag. Als „Erfolg“ konnte sie ihren Sympathisanten diese Propagandaoffensive schwer verkaufen, denn auf das Interesse der Passanten stieß sie nur in Ausnahmefällen. In Greifswald allerdings konnte sie nahezu ungestört ihre Propagandamaterialien an die Frau oder den Mann bringen.

Die NPD verfügt in Mecklenburg-Vorpommern über einen festen Aktivistenstamm von 400 Personen. ${ }^{33}$ Sie ist - im Gegensatz zu den anderen Landesverbänden - keiner hohen

31 Vgl. Andrea Röpke, Radikaler Flügel der NPD-Jugend, in: Blick nach Rechts, http://www.bnr.de/ artikel/hintergrund/radikaler-fluegel-der-npd-jugend (Abruf am 1. März 2013).

32 Vgl. Andreas Speit, Zeichen gesetzt, in: taz online vom 1. Mai 2012, http://www.taz.de/!92552/ (Abruf am 22. Februar 2013).

33 Vgl. Ministerium für Inneres und Sport (Hrsg.), Verfassungsschutzbericht 2011, Schwerin 2012, S. 15. 
Fluktuation unterworfen. Seit einigen Jahren bewegen sich ihre Mitgliederzahlen um die 400. Selbst ihr Einzug in den Landtag 2006 beziehungsweise ihr Wiedereinzug 2011 löste keinen „Mitgliederboom“ aus. Offensichtlich verficht die Parteispitze um Landeschef Köster das Konzept einer Kaderorganisation. Nur diejenigen Interessenten, die nach Einschätzung der Führungspersonen für die politische Arbeit brauchbar sind, finden tatsächlich Aufnahme. Ihr Sympathisantenumfeld ist ungleich größer, was nicht zuletzt ihre Wahlresultate zeigen.

Mit Nachdruck trieb die NPD ihre gesellschaftliche Verwurzelung in ihren Schwerpunktregionen voran. In Lübtheen, dem Heimatort Udo Pastörs, bezog sie ein neues Bürgerbüro in zentraler Lage, das durch Parteiaufkleber und Plakate bereits von Weitem als NPD-Anlaufstelle zu erkennen ist. Die Wahlkreismitarbeiter von Pastörs und Köster, Andreas Theißen und Torgai Klingebiel, nutzen den Stützpunkt, um das antidemokratische NPD-Gedankengut weiter in die Bevölkerung zu tragen. Nur in wenigen Regionen Deutschlands kann die Partei ihr Selbstbewusstsein so offen zur Schau stellen wie in Lübtheen. Hier muss die NPD nicht erst in die „Mitte der Gesellschaft“ vorstoßen, sie ist dort schon lange angekommen. Bereits vor seinem Landtagseinzug war der Uhrmachermeister Pastörs in dem 4.500-Einwohner-Städtchen ein geachteter Mann. Ende der 1990er Jahre war der Rechtsextremist von Nordrhein-Westfalen nach Lübtheen gezogen, um dort die „Keimzelle einer neuen nationalen Bewegung" zu schaffen. ${ }^{34}$ Die Bemühungen tragen Früchte; in den letzten Jahren siedelten sich einige Familien mit entsprechender Gesinnung im ehemaligen Landkreis Ludwigslust an. ${ }^{35}$

In unmittelbarer Nähe des Parteibüros eröffnete im Frühjahr 2012 im ehemaligen „Hotel Stadt Hamburg“ ein „Kulturraum“, in dessen Veranstaltungskalender neben unpolitischen Angeboten wie „Handarbeitstreffen“ oder „Spieleabende“ ebenfalls „politische Diskussionsrunden“ stehen. Obwohl die NPD nach außen nicht als Veranstalterin auftritt, bestehen keine Zweifel an ihrem Einfluss: Zu den Referenten gehörten der sächsische NPDAbgeordnete Arne Schimmer, der über „Europa am Abgrund! Eurokrise und Finanzdiktatur“ sprach, der einstige Chef der verbotenen neonationalsozialistischen „Wiking Jugend“ (WJ), Wolfram Nahrath, genauso wie der bekannte Holocaust-Leugner Rigolf Henning.

\section{Kinderfeste als "Türöffner"}

Nach wie vor benutzen die NPD-Strategen vermeintlich nicht-politische Angebote als „Türöffner", um mit den Menschen in Mecklenburg-Vorpommern in Kontakt zu kommen: Mit „Kinderfesten“ möchte die Partei zuerst die Kinder, und dann deren Eltern an sich binden. Während sich die Mädchen und Jungen auf einer Hüpfburg austoben, mit leuchtenden Augen den Darbietungen von Clown „Maex“ folgen oder ihnen NPD-Unterstützer lustige Tierfiguren auf die Gesichter malen, stärken sich die Erwachsenen mit kostenlosen Würst-

34 Vgl. Philipp Wittrock, Der Siegeszug der braunen Sieder, in: Spiegel Online vom 26. September 2006, http://www.spiegel.de/politik/deutschland/npd-basis-luebtheen-siegeszug-der-braunensiedler-a-438707.html (Abruf am 1. Oktober 2012).

35 Vgl. Andrea Röpke / Andreas Speit, Völkisch-radikale Enklave im Nordosten, in: Blick nach Rechts vom 16. Juli 2012, http://www.bnr.de/artikel/hintergrund/voelkisch-radikale-enklave-im-nordosten (Abruf am 3. Oktober 2012). 
chen, Kuchen und Kaffee. Ganz nebenbei - wie zufällig - verwickeln die anwesenden Parteifunktionäre die Gäste in Gespräche und bringen ihre Weltanschauung unters Volk.

Dafür nutzen die NPD-Kader alle landesweiten „Leuchttürme“. Selbst ins „Thinghaus“, einem beliebten - und mit Wachturm und Palisadenzaun gut befestigten - Szenetreffpunkt in Grevesmühlen (Landkreis Nordwestmecklenburg) mit Ausstrahlung weit über die Grenzen der Gemeinde hinaus, werden Interessierte zum „Sehen, Reden, Kennenlernen“ eingeladen - sozusagen Kinderfeste hinter Stacheldraht. Das „Thinghaus“ gehört zu den wichtigsten Logistikzentren der Nordost-NPD. ${ }^{36}$ In dem Gebäude am Stadtrand, das dem ehemaligen NPD-Landesvorstandsmitglied und wegen gewerbsmäßiger Hehlerei und illegalen Waffenbesitzes vorbestraften Sven Krüger gehört, bieten Pastörs und Köster Sprechstunden in ihren Bürgerbüros an. Außerdem hat das von David Petereit verantwortete Internetportal „MUPinfo“ hier seinen Sitz. Gleiches gilt für die Initiative „Netzwerk für Tolerie und Demokranz“, hinter der ebenfalls Petereit steckt, und mit der die Landesprogramme für Demokratie und gegen Rechtsextremismus verunglimpft werden sollen. Obwohl mit dicken Lettern „Willkommen“ auf dem in Grevesmühlen verteilten Flyer prangt, gilt dies nicht für jeden: „Personen, die antideutschen Parteien oder Organisationen angehören, der linksextremen Szene zuzuordnen sind, oder bereits in der Vergangenheit durch antinationale, linksextreme oder sonstige menschenverachtende Äußerungen in Erscheinung getreten sind“, würden die Veranstalter den Zugang verweigern, heißt es dort.

Zusätzlich setzt die NPD weiterhin auf Informationszeitungen, die auf den ersten Blick nicht der Partei direkt zugeordnet werden sollen. Trat vormals der Verein „Initiative für Volksaufklärung“ als Herausgeber dieser NPD-Tarnblättchen auf, werden sie nun presserechtlich von lokalen Funktionären verantwortet. In mehr oder weniger regelmäßigen Abständen verbreiten die Verfasser in verschiedenen Regionalausgaben Nachrichten aus der NPD-Fraktion oder der Landespartei. Lokale Themen greifen die „Boten“ gern auf, oft sind auch Aufforderungen an die Leser enthalten, selbst aktiv zu werden, mit der NPD in Kontakt zu treten oder sich direkt dieser anzuschließen. Ein Terminkalender mit Hinweisen zu Veranstaltungen der Partei oder ihr nahestehender Organisationen runden die rechtsextremistischen Propaganda-Postillen ab. 2012 tauchte in Schwerin zum ersten Mal ein entsprechendes Pamphlet, der „Meckelbörger Bote“, auf.

\section{Ungleiche Partner: Die Putschisten aus Sachsen und Mecklenburg-Vorpommern}

Unterdessen konnte die Landes-NPD ihre Stellung in der Bundespartei ausbauen. Hatte der Tod des ehemaligen Kopfes des radikalen Parteiflügels, Jürgen Rieger, den Landesverband vorübergehend ins Abseits geführt, da sich der angeschlagene einstige Bundeschef Udo Voigt wieder seinem Ziehsohn Holger Apfel annäherte, schlug nun die Stunde von Udo Pastörs und seinen Anhängern. Seit bekannt geworden war, dass der ehemalige NPD-Schatzmeister und langjährige Weggefährte Voigts, Erwin Kemna, rund 750.000 Euro aus der Parteikasse veruntreut hatte, blies Voigt der Wind heftig ins Gesicht. Dabei war er es, der die NPD mit seiner organisatorischen, ideologischen und strategischen Neuausrichtung zurück in die (begrenzte) Erfolgsspur geführt hatte. In seiner 15-jährigen Amtszeit prägte er die Partei wie

36 Vgl. Roman Heflik, Grevesmühlen ist die Festung der Extremisten, in: Hamburger Abendblatt vom 29. August 2011. 
nur ein Vorsitzender vor ihm, der charismatische Adolf von Thadden (1967 bis 1971). ${ }^{37}$ In der Endabrechnung zählten die Verdienste des „Kameraden“ nicht: Die parteiinterne Opposition hielt ihm seine Verstrickung in die „Affäre Kemna“ vor, außerdem hatte er in den letzten Jahren politische Visionen vermissen lassen. Auch seine Wahlergebnisse blieben hinter den Erwartungen zurück. Die von Voigt geführte NPD erreichte bei der Abgeordnetenhauswahl in Berlin 2011 nur 2,6 Prozent; mit ihrer provokanten Wahlkampfstrategie, die in einem perfiden Plakat mit dem Slogan „Gas geben!“ gipfelte, reüssierte sie nicht. Die Gegner Voigts in den Landesverbänden Sachsen und Mecklenburg-Vorpommern feierten unterdessen den Wiedereinzug in das jeweilige Parlament, folgenreiche Punktsiege, denen der einstige NPD-Vorzeigestratege nichts entgegenzusetzen hatte.

Bereits 2009 hatten Apfel und Pastörs einen ersten Griff nach dem NPD-Chefsessel gewagt. Als designierten Herausforderer hatten sie zunächst den Parteivordenker Andreas Molau, der Mitte August 2012 medienwirksam seinen Ausstieg aus der rechtsextremistischen Szene verkündete ${ }^{38}$, in den Ring geschickt. Doch Molau, der damals als Pressesprecher für die Schweriner Fraktion arbeitete, verfolgte eigene Visionen; er wollte der Partei einen europäischen Nationalismus verordnen, was an der mehrheitlich neonationalsozialistsch ausgerichteten Basis heftigen Widerstand hervorrief. ${ }^{39}$ Die offene Flanke schlossen die Putschisten, indem sie dem einstigen Waldorfschullehrer Molau das Vertrauen entzogen, woraufhin dieser die NPD verließ - nicht ohne kräftig nachzutreten. Die Rolle des Kandidaten zum Parteivorsitzenden spielte fortan der Hardliner Pastörs, der sich auf dem Berliner Parteitag als Retter vor einer vermeintlichen „Verbürgerlichung“ der NPD zu inszenieren wusste. Einen Stich machte er damit indes nicht: Im Verbund mit dem „radikaleren“ Parteiflügel, als dessen Führungsfigur gemeinhin Rieger galt, hatte Voigt seine Reihen frühzeitig schließen können.

\section{8. "Seriöse Radikalität“-Nicht in Mecklenburg-Vorpommern}

In Sachsen legte die Partei früh die Grundsteine für ihren „Zweiten Frühling“ “40. Die Ansiedlung der parteieigenen Infrastruktur im Freistaat, der Zuzug hoffnungsvoller Kader aus den westdeutschen Bundesländern (Holger Apfel, Alexander Delle, Jürgen Gansel) und die Gewinnung einiger regional angesehener Lokalpersönlichkeiten wie Uwe Leichsenring oder Johannes Müller sicherten der Gliederung eine Sonderstellung, die bis heute anhält. ${ }^{41} \mathrm{Im}$ Schatten dieser „Erfolgsgeschichte“ entwickelte sich - weitgehend unbemerkt - die NPD Mecklenburg-Vorpommern zum elektoral erfolgreichsten Landesverband. Während die Sachsen in-

37 Vgl. Marc Brandstetter, Die NPD unter Udo Voigt. Organisation, Ideologie, Strategie, BadenBaden 2013.

38 Vgl. Angelika Henkel/ Stefan Schölermann, Rechter Vordenker kehrt Szene den Rücken, in: NDR Info Online vom 30. Juli 2012, http://www.ndr.de/regional/dossiers/der_norden_schaut_hin/ molau101.html (Abruf am 3. Oktober 2012).

39 Vgl. Marc Brandstetter, Zerstritten, pleite, geächtet: Wohin führt der Weg der NPD?, in: Deutschland Archiv, 42. Jg. (2009), H. 3, S. 389 - 393.

40 Armin Pfahl-Traughber, Der „zweite Frühling“ der NPD. Entwicklung, Ideologie, Organisation und Strategie einer rechtsextremistischen Partei, St. Augustin / Berlin 2008.

41 Vgl. Henrik Steglich, Die NPD in Sachsen. Organisatorische Voraussetzungen ihres Wahlerfolges 2004, Göttingen 2005. 
nerhalb einer Wahlperiode fast die Hälfte ihrer ursprünglichen Wähler verloren und von 9,2 (2004) auf 5,6 Prozent (2009) abstürzten, fielen die Verluste an der Ostsee vergleichsweise gering aus. 2011 erreichte die NPD bei den Landtagswahlen 6,0 Prozent - stärker ist sie nirgends.

Dennoch bestimmen die sächsischen NPD-Spitzenfunktionäre die politische Linie der Partei auf Bundesebene. Mit der Wahl Apfels auf den Chefsessel im November 2011 entschied der „gemäßigtere“ Flügel den jahrelang schwelenden Konflikt über die strategische Ausrichtung (vorerst) zu seinen Gunsten. Dabei liegen die konkurrierenden Strömungen ideologisch nicht weit auseinander, beide einte und eint das Ziel, die parlamentarische Demokratie abzuschaffen. Aber über den Weg und die Mittel wird heftig gestritten. Apfel und seine Mitstreiter setzen auf das Konzept der „seriösen Radikalität“, sie verpassen der NPD eine „gemäßigtere“ Außendarstellung, um neue Wählerschichten zu erschließen und nicht zu einer „Polit-Sekte und Bürgerschrecktruppe“ zu verkommen. Die Botschaft müsse heißen: „Aus dem Volk für das Volk. “42 Mit ihren ideologischen Grundfesten bricht die NPD aber nicht, das „Abstammungsprinzip“ und die Ideologie einer ethnisch homogenen Volksgemeinschaft bilden weiterhin die Säulen ihrer Weltanschauung. Die Spaltung der NPD ist damit keineswegs überwunden, im Gegenteil: Der vermeintlich „weichgespülte“ Kurs des Bundesvorsitzenden treibt die Hardliner auf die Barrikaden. In einigen Landesverbänden wie Bayern oder Sachsen verlassen sie scharenweise die Partei - zumal zählbare Erfolge ausbleiben. Weder konnte die NPD bei den ersten Landtagswahlen unter Apfels Führung „Achtungserfolge" feiern, noch konnte sie ihren Mitgliederschwund stoppen.

Die „Kameraden“ an der Ostsee kümmert die neue Marketingstrategie der Bundesspitze wenig. Hier ist der Schulterschluss zwischen klassischen Parteikadern und radikalen Neonazis fester denn je. Die NPD ist stramm neonationalsozialistisch ausgerichtet, die einzelnen Flügel der „Bewegung“ lassen sich nicht auseinanderdividieren. Dafür wagt die Führungsetage der Landespartei einen Spagat: Auf Bundesebene hat sie mit den „gemäßigteren“ Parteianhängern ein fragiles Kampfbündnis geschmiedet, dessen Zweck einzig und allein die Ablösung Udo Voigts war. Zog man einst noch gemeinsam an einem Strang, könnte diese Eintracht bald vorbei sein. Längst verbreiten sich in der Partei Gerüchte, dass Pastörs mit der Unterstützung der neonationalsozialistischen Kräfte, die an der Basis bundesweit nach wie vor die Mehrheit stellen, am Stuhl des angezählten Apfel sägt.

Auch Udo Voigt wagt sich angesichts der verbesserten Ausgangssituation wieder aus der Deckung. Enttäuschte (ehemalige) NPD-Sympathisanten sammeln sich in „Freundeskreisen“, die den Namen des langjährigen Parteichefs tragen. ${ }^{43}$ Der betont zwar, man wolle „nicht spalten und schwächen“, stellt der „seriösen Radikalität“ seines Nachfolgers Apfel aber eine Alternative entgegen: die Zusammenarbeit aller wohlwollenden Flügel des „nationalen Widerstandes“. „Es hat fast zehn Jahre gedauert eine ,Volksfront' von Rechts zumindest in Ansätzen zu formen. Dieser Gedanke darf nicht leichtfertig und konzeptionslos aufgegeben werden", kritisiert das Parteiurgestein auf Facebook - eine Kampfansage, die der einstige Bundeswehroffizier so aber nicht verstanden wissen will. Trotzdem: Holger Apfel ist wie die gesamte NPD schwer angezählt.

42 Holger Apfel, „Seriöse Radikalität“, in: Deutsche Stimme, 36. Jg. (2011), H. 11, S. 3.

43 Vgl. „Organisierter Wille bedeutet Macht. Neugegründete ,Freundeskreise Udo Voigt' vereinen deutsch-nationale Kräfte“, 30. Januar 2013, http://wohin-deutscherechte.de/?p=1 (Abruf am 1. März 2013). 